\title{
Salivary levels of MPO, MMP-8 and TIMP-1 are associated with gingival inflammation response patterns during experimental gingivitis
}

\author{
Gustavo G. Nascimento ${ }^{\mathrm{a}, *}$, Vibeke Baelum ${ }^{\mathrm{b}}$, Timo Sorsa ${ }^{\mathrm{c}, \mathrm{d}}$, Taina Tervahartiala ${ }^{\mathrm{c}}$, \\ Peter D. Skottrup ${ }^{\mathrm{e}, \mathrm{f}}$, Rodrigo López \\ ${ }^{a}$ Section of Periodontology, Department of Dentistry and Oral Health, Aarhus University, Aarhus, Denmark \\ ${ }^{\mathrm{b}}$ Section of Oral Epidemiology and Public Health, Department of Dentistry and Oral Health, Aarhus University, Aarhus, Denmark \\ ${ }^{\mathrm{c}}$ Department of Oral and Maxillofacial Diseases, University of Helsinki and Helsinki University Central Hospital, Helsinki, Finland \\ ${ }^{\mathrm{d}}$ Department of Dental Medicine, Karolinska Institutet, Huddinge, Sweden \\ ${ }^{\mathrm{e}}$ Department of Clinical Biochemistry, Copenhagen University Hospital, Hvidovre DK-2650, Hvidovre, Denmark \\ ${ }^{\mathrm{f}}$ Novo Nordisk A/S, Research Bioanalysis, Global Research Technologies, DK-2760 Måløv, Denmark
}

\section{A R T I C L E I N F O}

\section{Keywords:}

Immunology

Innate immunity

Neutrophils

Gingival inflammation

Periodontal diseases

\begin{abstract}
A B S T R A C T
Aim: This study aimed to investigate the association between salivary levels of myeloperoxidase (MPO), neutrophil elastase (NE), soluble urokinase-type plasminogen activator receptor (suPAR), matrix metalloproteinase (MMP)-8 and tissue inhibitor of matrix metalloproteinases (TIMP)-1 and gingival inflammation development during an experimental gingivitis study.

Methods: A three-week experimental gingivitis study was conducted. Clinical recordings of dental plaque biofilm (Modified Quigley Hein Plaque Index, TQHPI) and gingival inflammation (Modified Gingival Index, MGI) were made at specific time points for each of the 42 participants. Salivary levels of MPO, NE, suPAR, MMP-8 and TIMP-1 at the same time points were measured using distinct immunoassays. For data analysis growth curve modelling was employed to account for the time-varying outcome (MGI score) and the time-varying covariates (salivary marker levels, and TQHPI score). Analyses were stratified according to the MGI-score trajectory groups previously identified as 'fast', respectively 'slow' responders.

Results: Overall, higher MGI scores were statistically significantly positively associated with higher levels of MPO, MMP-8 and TIMP-1. Stratified analysis according to inflammation development trajectory group revealed higher levels of salivary MPO, MMP-8 and MMP-8/TIMP-1 ratio among the 'fast' responders than among 'slow' responders. None of the investigated salivary protein markers was associated with a 'slow' inflammation development response.

Conclusions: Salivary levels of MPO, MMP-8 and TIMP-1 were associated with the extent and severity of gingival inflammation. While the 'fast' gingival inflammation response was associated with increased levels of MPO, MMP-8 and MMP-8/TIMP-1 ratio, the 'slow' response was not associated with any of the salivary protein markers investigated in this study. Neutrophil activity seems to orchestrate a 'fast' gingival inflammatory response among participants previously primed to gingival inflammation.
\end{abstract}

\section{Introduction}

Current understanding of the etiopathogenesis of periodontal diseases recognizes the host immune response as a key factor in the onset and progression of periodontal diseases [1]. In essence, a host immuneinflammatory response is elicited as a response to dysbiotic plaque accumulation, with local proinflammatory cytokines and chemokines attracting neutrophils to the infection site [2]. In an attempt to control tissue invasion by bacteria, neutrophils release myeloperoxidase (MPO) and elastase (NE), which in turn activate matrix metalloproteinases (MMPs) [3]. Local activation of MMPs may lead to periodontal destruction if the MMP is not inactivated by specific regulators, such as the tissue inhibitor of matrix metalloproteinases (TIMP)-1 [4]. In periodontal research, MMP-8 is one of the most frequently investigated MMPs, since the MMP-8/TIMP-1 relationship is closely related to periodontal tissue destruction [5]. Stimulated neutrophils also release

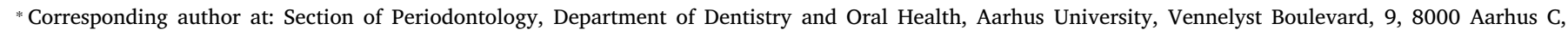
Denmark.

E-mail address: ggn@dent.au.dk (G.G. Nascimento). 
suPAR (soluble urokinase-type Plasminogen Activator Receptor), a chemotactic agent related to the recruitment of other immune cells to the inflamed site [6].

While many attempts have been made to identify distinct response patterns in gingivitis development, most of these present methodological caveats, such as neglect of the data structure and the longitudinal study design, what has precluded a deeper understanding of the factors involved in the health-gingivitis dynamics [7]. In a recent experimental gingivitis study, we were able to identify two different patterns of gingival inflammation development dubbed 'fast' and 'slow' response [8]. Whilst the 'fast' responders seemed capable of responding rapidly to biofilm accumulation, the 'slow' responders presented a lagged development of gingival inflammation for the same biofilm insult [8].

Even though individual variation in the susceptibility to develop gingivitis has been intensively discussed, the mechanisms involved in this process remain unclear. Previous observations of individual variation in the gingival response patterns to experimental plaque accumulation have loosely been attributed to differences in "individual subject's resistance" [9], but no further investigation has been carried out of the role played by the immune response. The study of key protein markers involved in the inflammatory response may therefore be helpful in clarifying the different patterns of gingival inflammation responses to experimental plaque accumulation. For that reason, the present study aimed to investigate the association between salivary levels of MPO, NE, suPAR, MMP-8 and TIMP-1 and the patterns of development of gingival inflammation during a period of experimental plaque accumulation.

\section{Methods}

\subsection{Experimental gingivitis study}

Participants for this experimental gingivitis study were recruited among students of Aarhus University. Eligibility criteria included age between 18 and 35 years, a minimum of 20 teeth present, no pocket probing depth $>4 \mathrm{~mm}$, and no evidence of interproximal clinical attachment loss $>2 \mathrm{~mm}$. Individuals were excluded if they had used antibiotics or anti-inflammatory medications within the previous six weeks, had systemic conditions interfering with the immune system, or had retentive factors for plaque in the form of orthodontic appliances, crowns, bridgework, or inadequate restorations. Exclusion criteria also included pregnancy (current or planned), breastfeeding, or any impairment that precluded effective personal oral hygiene.

Participation was based on written informed consent and the study was approved by the Ethical Committee of the Central Denmark Region (j.nr. 1-10-72-402-14). Volunteers who agreed to participate in the study were invited to a screening visit that comprised clinical examinations and information collected with the use of a questionnaire. This visit aimed to evaluate the individuals' eligibility for the experimental phases of the study. Self-reported information on smoking was also gathered and validation of the self-reported smoking status information was done by quantifying salivary cotinine using a high sensitivity enzyme-linked immunosorbent assay (ELISA) assay (Salimetrics Europe Ltd., Newmarket, UK).

The study comprised three phases: A two-week run-in Hygiene Program Phase; a three-week experimental plaque accumulation Induction Phase; and a two-week Resolution Phase. Individuals who fulfilled the eligibility criteria were enrolled in the two-week Hygiene Program. This program aimed to reduce plaque and calculus deposits and gingival inflammation prior to the Induction Phase. The program comprised at least two visits, in which plaque was disclosed, supra- and subgingival plaque and calculus removed, and oral hygiene instructions provided. Throughout the Hygiene Program, all participants were instructed to perform oral hygiene twice daily with standard toothbrush and toothpaste provided by the researchers. Individuals were permitted to proceed to the Induction Phase when they had achieved bleeding on probing $<4 \%$ on all units examined and visible plaque $<20 \%$ of all units examined.

During the Induction Phase, the participants were instructed to abstain from all oral hygiene procedures for three weeks. Participants were specifically asked not to use other oral hygiene products, including chewing gum, mouth rinse solutions or dental floss. When the three-week experimental Induction Phase was completed, all participants were supplied with a toothbrush and toothpaste to reestablish self-performed oral hygiene. At the end of the two-week Resolution Phase, after examinations have been conducted, a professional cleaning was provided to participants who required additional care to restore gingival health.

The Modified Quigley Hein Plaque Index (TQHPI) was used for plaque recordings [10], and the Modified Gingival Index (MGI) was used for clinical recordings of gingival inflammation [11]. Plaque was recorded in the papillary and marginal units of each tooth from the buccal aspect, leading to a maximum of 56 recordings per participant; while recordings of gingival inflammation were also done from the lingual/palatal aspect, leading to a maximum of 112 recordings per participant. Clinical recordings were performed at days $0,7,14,21$ and 35 .

\subsection{Saliva sampling}

All saliva samples were collected in the morning between $8 \mathrm{AM}$ and 10 AM. Participants were asked to avoid eating, drinking and performing oral hygiene for two hours before the visit. Prior to the saliva collection, participants rinsed their mouth with water twice, and waited five minutes after rinsing to avoid saliva dilution. Saliva was obtained by passive drooling for five minutes in a sterile $50 \mathrm{~mL}$ polypropylene conic tube. Saliva was aliquoted, stored, and frozen at $-80^{\circ} \mathrm{C}$ (for suPAR analyses) or at $-20^{\circ} \mathrm{C}$ (for all other markers).

\subsection{Neutrophil elastase, MPO, TIMP-1 and MMP-8 assessment}

The concentrations of salivary neutrophil elastase, MPO and TIMP-1 were determined by commercially available enzyme-linked immunosorbent assay (ELISA) kits according to the manufacturer's protocol as described by Akcali et al. [12]. The detection limit was $1.98 \mathrm{pg} /$ $\mathrm{mL}$ using human Neutrophil Elastase Platinum ELISA (Bender MedSystems, Vienna, Austria), $1.6 \mathrm{ng} / \mathrm{mL}$ using MPO ELISA Kit (Immundiagnostik, Bensheim, Germany), and $1.25 \mathrm{ng} / \mathrm{mL}$ using Amersham TIMP-1, human Biotrak ELISA system (Amersham Biosciences, Little Chalfont, UK). The saliva MMP-8 levels were analysed by time-resolved immunofluorometric assay (IFMA) (Medix Biochemica, Espoo, Finland) with the detection limit of $0.08 \mathrm{ng} / \mathrm{mL}$. The molar ratios of MMP-8/ TIMP-1 were calculated and converted to mol/L [13].

\section{4. sUPAR assessment}

Concentrations of salivary suPAR were measured in duplicate using an automated ELISA robot (suPARnostic AUTO Flex Kit, Lot. No. 204LA1-1; Virogates, Birkerød, Denmark). The assay detection limit was $1 \mathrm{ng} / \mathrm{mL}$. Prior to analysis, samples were centrifuged at $5000 \mathrm{~g}$ for $10 \mathrm{~min}$ at $4{ }^{\circ} \mathrm{C}$, and then diluted $1: 1$ in assay dilution buffer.

\subsection{Data analysis}

Individual mean TQHPI and MGI scores were calculated for each time point of the study. Group-based-trajectory-modelling was applied to model patterns of development in MGI scores over time during the three-week Induction Phase [14]. This analytical approach allowed us to identify two groups of participants who follow distinctly different patterns of gingival inflammation development under the study period; one group dubbed 'slow' responders, and one dubbed 'fast' responders. Detailed information on this analysis can be found elsewhere [8]. 

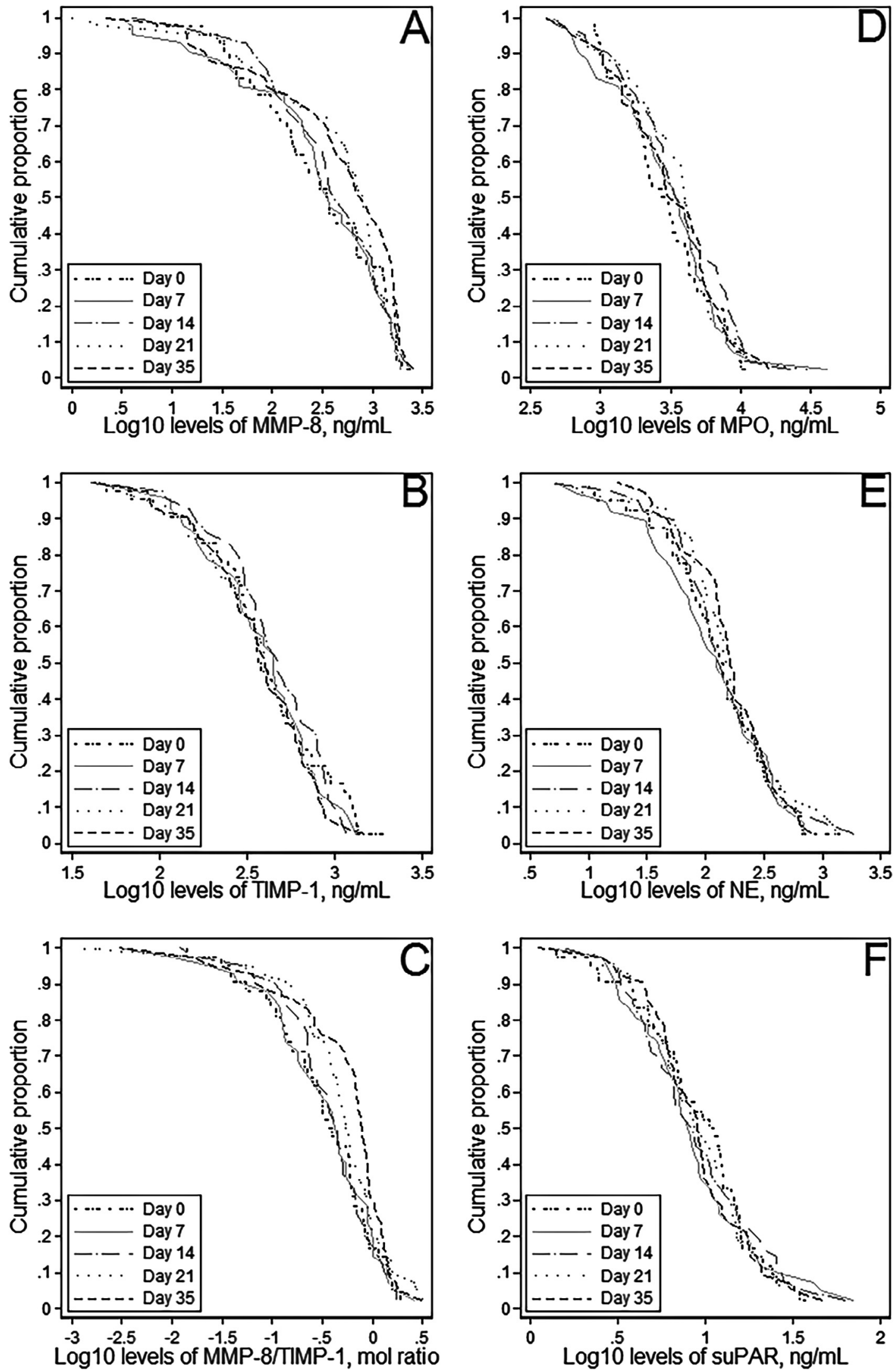

Fig. 1. $\log _{10}$ transformed levels of (A) MMP-8, ng/ml; (B) TIMP-1, ng/ml; (C) MMP-8/TIMP-1 ratio, mol ratio; (D) MPO, ng/ml; (E) NE, ng/ml; (F) suPAR, ng/ml. 
Table 1

Mean values (SD) of the scores for the clinical parameters TQHPI and MGI, and of the concentrations of the salivary markers in the samples according to Day of Experiment. Given for the total study group $(n=42)$.

\begin{tabular}{|c|c|c|c|c|c|}
\hline & \multicolumn{5}{|c|}{ Day of experiment $(n=42)$} \\
\hline & 0 & 7 & 14 & 21 & 35 \\
\hline \multicolumn{6}{|l|}{ Clinical Data } \\
\hline \multirow[t]{2}{*}{ TQHPI score } & 0.14 & 1.59 & 2.52 & 3.01 & 0.57 \\
\hline & $(0.1)$ & $(0.5)$ & $(0.5)$ & $(0.4)$ & $(0.3)$ \\
\hline \multirow[t]{2}{*}{ MGI score } & 0.01 & 0.49 & 1.34 & 1.85 & 0.17 \\
\hline & $(0.0)$ & $(0.3)$ & $(0.4)$ & $(0.3)$ & $(0.2)$ \\
\hline \multicolumn{6}{|l|}{ Salivary Markers } \\
\hline \multicolumn{6}{|l|}{ Elastase } \\
\hline (ng/mL) & $(260.3)$ & $(182.2)$ & $(335.5)$ & (313.9) & $(185.4)$ \\
\hline MPO & 3808.8 & 4529.6 & 4771.2 & 4590.0 & 4223.2 \\
\hline (ng/mL) & $(2854.3)$ & $(6389.1)$ & (4178.5) & (4763.7) & $(3490.7)$ \\
\hline suPAR & 12.0 & 11.8 & 12.0 & 10.7 & 11.0 \\
\hline (ng/ml) & $(8.7)$ & $(13.0)$ & $(11.6)$ & $(6.9)$ & $(8.7)$ \\
\hline MMP-8 & 687.7 & 616.9 & 677.2 & 736.5 & 897.0 \\
\hline$(\mathrm{ng} / \mathrm{mL})$ & (704.7) & $(602.8)$ & $(652.8)$ & (579.1) & $(744.3)$ \\
\hline TIMP-1 & 542.8 & 479.6 & 525.1 & 459.6 & 451.6 \\
\hline (ng/mL) & $(430.0)$ & $(326.6)$ & (307.9) & $(303.0)$ & $(300.2)$ \\
\hline MMP-8/TIMP-1 & 0.57 & 0.56 & 0.54 & 0.73 & 0.80 \\
\hline (mol ratio) & $(0.5)$ & $(0.5)$ & $(0.5)$ & $(0.7)$ & $(0.6)$ \\
\hline
\end{tabular}

The data set available for analysis consisted of data on salivary protein markers, gingival inflammation (MGI) scores and dental plaque biofilm (TQHPI) scores for each of the time points day 0, 7, 14, and 21. Such data lend themselves to execute growth curve modelling as multilevel mixed-effects regression analysis at individual level, in order to account for the time-varying clinical outcome (the MGI score) and the time-varying covariates (salivary marker levels, TQHPI score) being nested within each individual. Levels of salivary protein markers were $\log _{10}$ transformed due to non-normal distribution and over-dispersion (Fig. 1). In order to allow comparability between salivary protein markers and MGI score, all variables were standardized to a mean value of 0 and a standard deviation of 1 .

Initially, we attempted to run a single model simultaneously including all salivary protein markers as independent variables. However, the large standard errors observed indicated model misspecification, and given the sample size studied and the resulting lack of degrees of freedom, we therefore decided to carry out analytical models using one marker at a time. Multilevel analyses were adjusted for plaque accumulation and stratified by 'fast' and 'slow' gingival inflammation response.

\section{Results}

Forty-two participants [64.3\% females, mean age $23.8 \mathrm{yr}$ (SD $3.6 \mathrm{yr})]$ were enrolled and completed all three-study phases. None of the participants reported smoking, and this was confirmed by the salivary cotinine ELISA assay results (data not shown). Table 1 summarizes the clinical data and the salivary protein marker observations for all participants. TQHPI and MGI scores increased gradually and continually over the Induction Phase (days 0, 7, 14 and 21) and had returned to much lower levels at the end of the Resolution Phase (day 35). As evidenced by the comparatively large standard deviations of the observations for all six protein markers considered, the distributions showed pronounced skewness, which led us to stratify the data by the previously identified inflammatory development pattern (Table 2). Evidently, 'fast' responders presented higher salivary levels of all markers than did 'slow' responders. However, although some reduction in the standard deviations was seen, skewness was still apparent, and this led us to $\log _{10}$ transform these data (Fig. 1) prior to further analysis.

The results of multilevel modelling of the effect of time and the standardized TQHPI score on the standardized MGI score are presented in Table 3, both for the overall sample and for the two inflammatory development pattern groups.

When salivary protein marker levels were added to the mixed-effects multilevel models presented in Table 3, the coefficients shown in Table 4 revealed that overall, increased levels of salivary myeloperoxidase, elastase, MMP-8 and TIMP-1 were associated with gingival inflammation (Table 4). However, analyses stratified according to the inflammatory development pattern showed that the overall contrasts were essentially attributable to the 'fast' responders, who had statistically higher levels of salivary myeloperoxidase, MMP-8 and MMP-8/ TIMP-1 molar ratio during the Induction Phase, where this was not the case for the 'slow' responders. None of the investigated protein markers was associated with the 'slow' response pattern (Table 4).

The predicted relationship between salivary protein markers and

Table 2

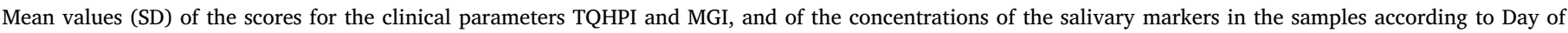
Experiment. Data presented according to the 'fast' and the 'slow' gingival inflammation development patterns.

Gingival inflammation development pattern

'Slow' responders $(\mathrm{n}=27) \quad$ 'Fast' responders $(\mathrm{n}=15)$

Day of experiment

Day of experiment

\begin{tabular}{|c|c|c|c|c|c|c|c|c|c|c|}
\hline & 0 & 7 & 14 & 21 & 35 & 0 & 7 & 14 & 21 & 35 \\
\hline \multicolumn{11}{|l|}{ Clinical Data } \\
\hline \multirow[t]{2}{*}{ TQHPI score } & 0.13 & 1.35 & 2.46 & 2.94 & 0.56 & 0.17 & 1.84 & 2.64 & 3.04 & 0.60 \\
\hline & $(0.1)$ & $(0.4)$ & $(0.5)$ & $(0.4)$ & $(0.3)$ & $(0.1)$ & $(0.4)$ & $(0.5)$ & $(0.3)$ & $(0.3)$ \\
\hline \multirow[t]{2}{*}{ MGI score } & 0.01 & 0.32 & 1.22 & 1.77 & 0.11 & 0.02 & 0.80 & 1.56 & 2.01 & 0.29 \\
\hline & $(0.0)$ & $(0.1)$ & $(0.3)$ & $(0.3)$ & $(0.1)$ & $(0.0)$ & $(0.2)$ & $(0.4)$ & $(0.2)$ & $(0.3)$ \\
\hline \multicolumn{11}{|l|}{ Salivary Markers } \\
\hline Elastase & 123.4 & 129.6 & 150.7 & 141.4 & 147.2 & 371.0 & 220.5 & 351.9 & 444.0 & 293.9 \\
\hline (ng/mL) & (125.9) & $(164.2)$ & (224.4) & (127.2) & $(142.7)$ & (356.1) & (203.5) & $(456.3)$ & $(445.3)$ & (217.3) \\
\hline MPO & 3088.3 & 4271.2 & 3669.2 & 3233.3 & 3558.7 & 5105.6 & 4994.5 & 6754.6 & 7032.1 & 5419.8 \\
\hline (ng/mL) & $(2434.8)$ & (7733.8) & (3127.6) & (1961.8) & $(3462.7)$ & (3169.7) & (2847.6) & (5138.9) & (7026.3) & (3321.9) \\
\hline suPAR & 9.4 & 9.6 & 9.7 & 8.7 & 9.2 & 16.6 & 15.8 & 16.3 & 14.3 & 14.4 \\
\hline (ng/ml) & (7.3) & (12.8) & $(9.0)$ & $(5.8)$ & (8.7) & (9.4) & (12.9) & (14.7) & (7.5) & $(7.8)$ \\
\hline \multirow[t]{2}{*}{ MMP-8 (ng/mL) } & 484.5 & 494.5 & 528.2 & 529.2 & 780.1 & 1053.3 & 837.3 & 945.5 & 1109.9 & 1107.6 \\
\hline & $(617.6)$ & $(557.0)$ & $(627.0)$ & $(466.3)$ & (776.9) & $(723.2)$ & $(638.0)$ & $(631.0)$ & $(588.2)$ & $(653.7)$ \\
\hline TIMP-1 & 403.3 & 372.5 & 416.6 & 385.7 & 379.5 & 793.9 & 672.4 & 720.4 & 592.6 & 581.2 \\
\hline (ng/mL) & (289.8) & (278.1) & (260.5) & (252.8) & (293.2) & (529.6) & (326.8) & (296.7) & $(347.4)$ & $(276.1)$ \\
\hline MMP-8/TIMP-1 & 0.55 & 0.57 & 0.55 & 0.59 & 0.80 & 0.60 & 0.54 & 0.54 & 0.97 & 0.81 \\
\hline (mol ratio) & $(0.6)$ & $(0.6)$ & $(0.5)$ & $(0.7)$ & $(0.7)$ & $(0.4)$ & $(0.4)$ & $(0.3)$ & $(0.8)$ & $(0.3)$ \\
\hline
\end{tabular}


Table 3

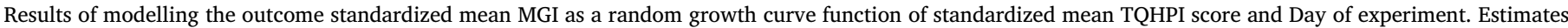
given are the regression coefficients with their respective 95\% confidence intervals (95\%CI). Results in bold indicate statistical significance.

\begin{tabular}{|c|c|c|c|c|}
\hline & & $\begin{array}{l}\text { Total Sample }(n=42) \\
\text { Coefficient }(95 \% \text { CI })\end{array}$ & $\begin{array}{l}\text { 'Slow' responders }(n=27) \\
\text { Coefficient }(95 \% \mathrm{CI})\end{array}$ & $\begin{array}{l}\text { 'Fast' responders }(\mathrm{n}=15) \\
\text { Coefficient }(95 \% \mathrm{CI})\end{array}$ \\
\hline \multirow[t]{4}{*}{ Induction Phase } & $\begin{array}{l}\text { Mean TQHPI score } \\
\text { Day (ref. day 0) }\end{array}$ & $0.55(0.37 ; 0.74)$ & $0.35(0.14 ; 0.55)$ & $0.60(0.37 ; 0.83)$ \\
\hline & Day 7 & $-0.02(-0.23 ; 0.15)$ & $0.04(-0.17 ; 0.25)$ & $0.15(0.00 ; 0.37)$ \\
\hline & Day 14 & $0.59(0.23 ; 0.96)$ & $0.88(0.42 ; 1.33)$ & $0.74(0.23 ; 1.25)$ \\
\hline & Day 21 & $1.03(0.57 ; 1.49)$ & $1.45(0.89 ; 2.02)$ & $1.10(0.54 ; 1.58)$ \\
\hline Resolution Phase & Day 35 & $0.00(-0.10 ; 0.10)$ & $0.00(-0.07 ; 0.07)$ & $0.11(-0.08 ; 0.32)$ \\
\hline
\end{tabular}

Table 4

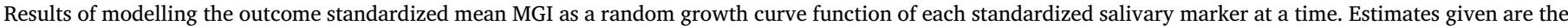

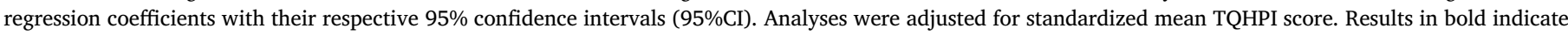
statistical significance.

\begin{tabular}{|c|c|c|c|}
\hline Salivary Markers & $\begin{array}{l}\text { Total Sample }(n=42) \\
\text { Coefficient }(95 \% \text { CI })\end{array}$ & $\begin{array}{l}\text { 'Slow' responders }(\mathrm{n}=27) \\
\text { Coefficient }(95 \% \mathrm{CI})\end{array}$ & $\begin{array}{l}\text { 'Fast' responders }(\mathrm{n}=15) \\
\text { Coefficient }(95 \% \mathrm{CI})\end{array}$ \\
\hline Myeloperoxidase & $0.09(0.00 ; 0.18)$ & $0.00(-0.09 ; 0.09)$ & $0.12(0.00 ; 0.24)$ \\
\hline Elastase & $0.05(0.00 ; 0.10)$ & $-0.01(-0.09 ; 0.08)$ & $0.05(-0.01 ; 0.10)$ \\
\hline suPAR & $0.02(-0.04 ; 0.08)$ & $-0.03(-0.11 ; 0.05)$ & $0.02(-0.06 ; 0.10)$ \\
\hline MMP-8 & $0.07(0.01 ; 0.14)$ & $0.04(-0.02 ; 0.12)$ & $0.07(0.00 ; 0.15)$ \\
\hline TIMP-1 & $0.06(0.01 ; 0.12)$ & $0.05(-0.01 ; 0.11)$ & $0.04(-0.03 ; 0.10)$ \\
\hline MMP-8/TIMP-1 ratio & $0.05(-0.02 ; 0.12)$ & $0.04(-0.02 ; 0.11)$ & $0.10(0.00 ; 0.20)$ \\
\hline
\end{tabular}

gingival inflammation at each time point of the Induction Phase is presented in Fig. 2. This figure shows that time was far more influential for the MGI score than was any of the salivary markers although weak positive correlations were observed for all markers except suPAR.

\section{Discussion}

The results of the present study have revealed an association between myeloperoxidase and MMP- 8 and the development of gingivitis in response to plaque accumulation. Neutrophil activity, expressed as levels of myeloperoxidase in saliva, was associated with a 'fast' clinical gingival response to plaque accumulation over the Induction Phase. Furthermore, an imbalance in the MMP-8/TIMP-1 molar ratio was also observed among the 'fast' responders, explained by increased levels of MMP-8. Conversely, the 'slow' response was not associated with any of the salivary markers under investigation in this study.

Leukocytes, especially neutrophils play a pivotal role in the orchestration of the inflammatory response. During the early phase of gingivitis development, neutrophils are the first line cells to perform phagocytosis in an attempt to neutralize the biofilm challenge [12]. Nevertheless, persistent neutrophil stimulation may lead to exacerbated production of inflammatory components, which may initiate tissue destruction [12]. A latent form of MMP-8 (proMMP-8) is released from secondary granules of neutrophils and other cells, such as plasma cells, fibroblasts and gingival sulcular epithelial cells [15]. Proinflammatory cytokines, neutrophil elastase and myeloperoxidase, among others, seem to induce and activate the latent MMP-8 [16]. While MPO stimulates the production of reactive oxygen species (ROS), which will in turn upregulate the activation of proMMP-8, and downregulate TIMP-1, neutrophil elastase directly activates proMMP-8 by proteolytic cleavage [17]. Neutrophils are also responsible for perpetuating the inflammatory response, because the insult cannot be readily eliminated. The production of elastase and suPAR by the neutrophils later in the inflammatory response is responsible for tissue remodelling and chemotaxis of other immune cells, respectively [6].

Host-derived inflammatory protein markers such as proinflammatory cytokines and enzymes, including myeloperoxidase, elastase and MMPs, are produced and accumulate as a response to plaque accumulation. Primed neutrophils tend to respond more rapidly when de novo exposed to plaque, being capable of instantly organizing an inflammatory reaction, which, in turn, may lead to early clinical signs of gingival inflammation [18]. We have shown that having higher mean scores of gingival inflammation at the day of screening for inclusion in this study was associated with a 'fast' response to plaque accumulation during the Induction Phase [8]. This indicates that the gingival inflammatory background might have primed the neutrophils. 'Fast' responders presented higher levels of all salivary markers at day 0 even after achieving a clinical situation of gingival health prior to the commencement of the Induction Phase (Table 1).

Our results showed that the levels of both MMP- 8 and TIMP-1 were positively associated with gingival inflammation in a similar magnitude, therefore, not changing the balance between MMP- 8 and its inhibitor. But as TIMP-1 inhibits all MMPs, MMP8 activity is most likely elevated, assuming that the levels of other MMPs remain constant. However, among the 'fast' responders, the results indicate that while the levels of MMP-8 increased as gingivitis developed, the levels of TIMP-1 did not follow the same course. This may explain the imbalance found in the MMP-8/TIMP-1 molar ratio noted among the 'fast' responders only. In a cross-sectional study, Noack et al. found that individuals with gingivitis presented higher salivary levels of MMP-8 than periodontally healthy individuals, but lower levels than found among those with periodontitis [19]. This indicates that MMP8 levels are closely related to the degree of tissue destruction. The increased levels of MMP-8 observed among the 'fast' responders may be a consequence of the increase in myeloperoxidase levels, which oxidatively upregulates the activation of proMMP-8 [4].

As expected, we did not find an association between gingival inflammation development and neutrophil elastase and suPAR. According to Uitto et al., elastase activity is not a reliable indicator of gingivitis [20]. Even though a peak of elastase is expected in the first days of gingivitis development, levels of elastase tend to decrease after few days of plaque accumulation [21]. In order to restrain the excessive self-damaging effect of elastase, alpha-1 protease inhibitor ( $\alpha 1 \mathrm{P} 1)$ and secretory leukocyte protease inhibitor (SLPI) are secreted in the saliva [22]. Nevertheless, increasing levels of elastase occur in periodontitis, therefore, reinforcing the role of this marker as an indicator of connective tissue destruction. Similarly, levels of suPAR are more pronounced in later - more chronic - stages of the inflammatory response, 

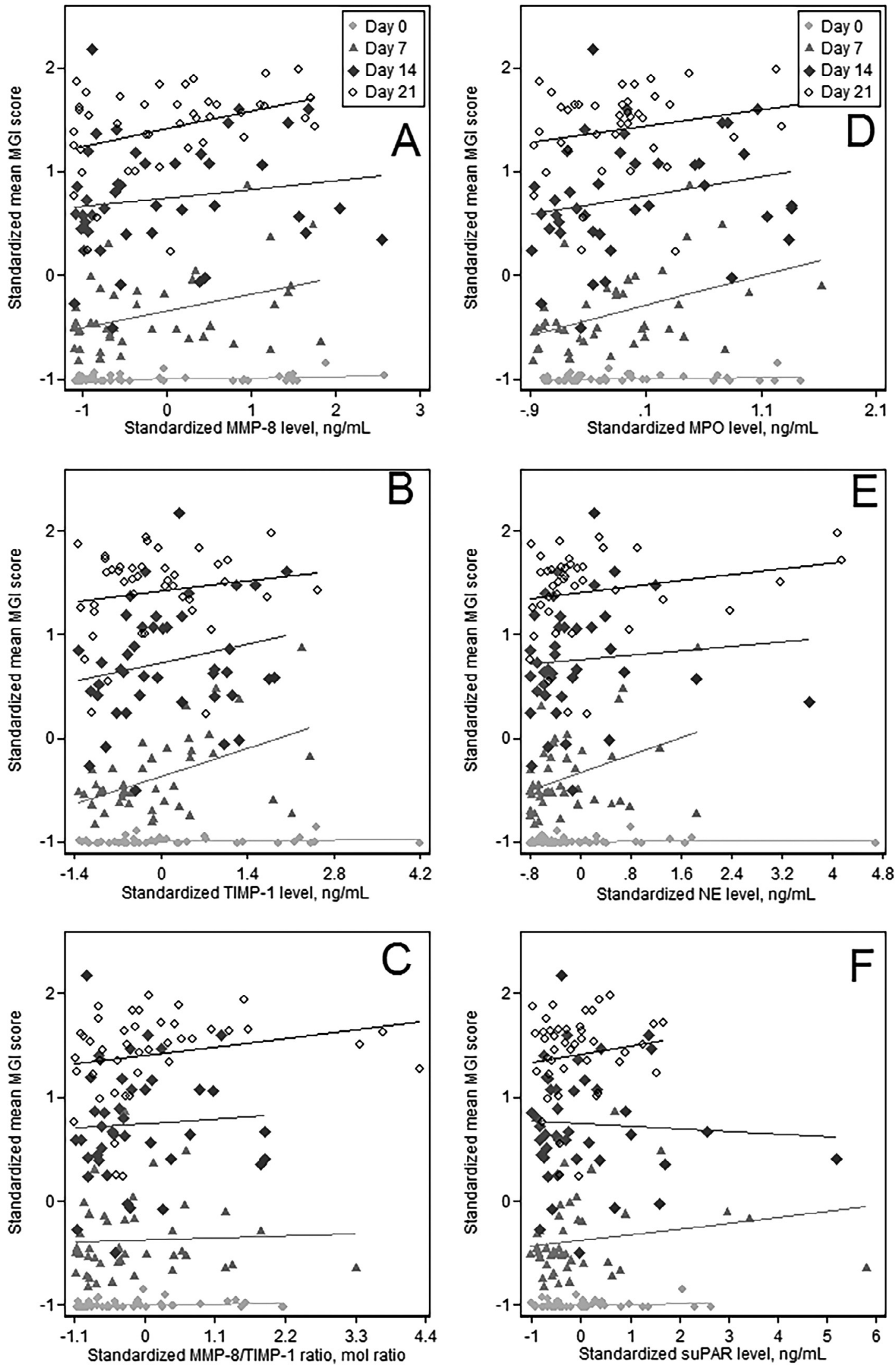

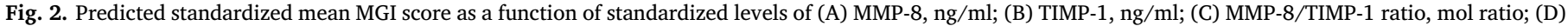
MPO, ng/ml; (E) NE, ng/ml; (F) suPAR, ng/ml. Predictive values are presented for the Induction Phase only. 
due to the chemotactic function of suPAR to attract other immune cells to the inflamed site. Such a function is more time-dependent, explaining why suPAR levels are higher in periodontitis cases. This is further supported by Skottrup et al. who found a positive association between suPAR and periodontitis among adolescents [23].

This study demonstrates that salivary levels of myeloperoxidase, and MMP- 8 and TIMP-1 are associated with gingival inflammation. However, a 'fast' gingival inflammation development to plaque accumulation was associated with increased levels of myeloperoxidase and the imbalance between MMP-8 and TIMP-1. The 'slow' response to plaque accumulation was not associated with any of the salivary protein markers investigated in this study. Overall, our results indicate that neutrophil activity orchestrates a 'fast' gingival inflammatory response among participants previously primed to gingival inflammation.

\section{Acknowledgments}

Philips Oral Healthcare, Philips AB donated electric toothbrushes to the participants at the end of the study, and Unilever HPC/Blumøller donated toothbrushes and toothpaste to cover consumption during the study.

\section{Funding}

The study was funded by Aarhus University Research Foundation, Denmark, and by strategic funds from Aarhus University, HEALTH, Denmark.

\section{References}

[1] G.G. Nascimento, F.R.M. Leite, F. Scheutz, R. López, Periodontitis: from Infection to Inflammation, Curr. Oral Health Rep. 4 (4) (2017) 301-308.

[2] A.E. Scott, M. Milward, G.J. Linden, J.B. Matthews, M.J. Carlile, F.T. Lundy, M.A. Naeeni, S. Lorraine Martin, B. Walker, D. Kinane, G.R. Brock, I.L. Chapple, Mapping biological to clinical phenotypes during the development (21 days) and resolution (21 days) of experimental gingivitis, J. Clin. Periodontol. 39 (2) (2012) 123-131.

[3] B.S. van der Veen, M.P. de Winther, P. Heeringa, Myeloperoxidase: molecular mechanisms of action and their relevance to human health and disease, Antioxid. Redox Signal. 11 (11) (2009) 2899-2937.

[4] T. Sorsa, L. Tjaderhane, Y.T. Konttinen, A. Lauhio, T. Salo, H.M. Lee, L.M. Golub, D.L. Brown, P. Mantyla, Matrix metalloproteinases: contribution to pathogenesis, diagnosis and treatment of periodontal inflammation, Ann. Med. 38 (5) (2006) 306-321.
[5] S. Izadi Borujeni, M. Mayer, P. Eickholz, Activated matrix metalloproteinase-8 in saliva as diagnostic test for periodontal disease? A case-control study, Med. Microbiol. Immunol. 204 (6) (2015) 665-672.

[6] B.K. Pliyev, M.Y. Menshikov, Release of the soluble urokinase-type plasminogen activator receptor (suPAR) by activated neutrophils in rheumatoid arthritis, Inflammation 33 (1) (2010) 1-9.

[7] L. Trombelli, D.N. Tatakis, C. Scapoli, S. Bottega, E. Orlandini, M. Tosi, Modulation of clinical expression of plaque-induced gingivitis. II. Identification of "high-responder" and "low-responder" subjects, J. Clin. Periodontol. 31 (4) (2004) 239-252.

[8] G.G. Nascimento, B. Danielsen, V. Baelum, R. Lopez, Identification of inflammatory response patterns in experimental gingivitis studies, Eur. J. Oral Sci. (2018), https://doi.org/10.1111/eos.12588 (in press).

[9] F. Abbas, U. Van der Velden, A.A. Hart, W.R. Moorer, T.M. Vroom, G. Scholte, Bleeding/plaque ratio and the development of gingival inflammation, J. Clin. Periodontol. 13 (8) (1986) 774-782.

[10] S. Turesky, N.D. Gilmore, I. Glickman, Reduced plaque formation by the chloromethyl analogue of victamine C, J. Periodontol. 41 (1) (1970) 41-43.

[11] R.R. Lobene, Discussion: Current status of indexes for measuring gingivitis, J. Clin. Periodontol. 13 (5) (1986) 2.

[12] A. Akcali, N. Bostanci, O. Ozcaka, P. Gumus, B. Ozturk-Ceyhan, T. Tervahartiala, H. Husu, N. Buduneli, T. Sorsa, G.N. Belibasakis, Gingival inflammation and salivary or serum granulocyte-secreted enzymes in patients with polycystic ovary syndrome, J. Periodontol. 88 (11) (2017) 1145-1152.

[13] H. Nagase, K. Brew, Designing TIMP (tissue inhibitor of metalloproteinases) variants that are selective metalloproteinase inhibitors, Biochem. Soc. Symp. 70 (2003) 201-212.

[14] B.L. Jones, D.S. Nagin, A note on a stata plugin for estimating group-based trajectory models, Sociol. Methods Res. 42 (4) (2013) 6.

[15] J.S. Kinney, C.A. Ramseier, W.V. Giannobile, Oral fluid-based biomarkers of alveolar bone loss in periodontitis, Ann. N. Y. Acad. Sci. 1098 (2007) 230-251.

[16] R. Romanelli, S. Mancini, C. Laschinger, C.M. Overall, J. Sodek, C.A. McCulloch, Activation of neutrophil collagenase in periodontitis, Infect. Immun. 67 (5) (1999) 2319-2326.

[17] M. Hernandez, J. Gamonal, T. Tervahartiala, P. Mantyla, O. Rivera, A. Dezerega, N. Dutzan, T. Sorsa, Associations between matrix metalloproteinase- 8 and -14 and myeloperoxidase in gingival crevicular fluid from subjects with progressive chronic periodontitis: a longitudinal study, J. Periodontol. 81 (11) (2010) 1644-1652.

[18] B. Amulic, C. Cazalet, G.L. Hayes, K.D. Metzler, A. Zychlinsky, Neutrophil function: from mechanisms to disease, Annu. Rev. Immunol. 30 (2012) 459-489.

[19] B. Noack, T. Kipping, T. Tervahartiala, T. Sorsa, T. Hoffmann, K. Lorenz, Association between serum and oral matrix metalloproteinase-8 levels and periodontal health status, J. Periodontal Res. 52 (5) (2017) 824-831.

[20] V.J. Uitto, A. Nieminen, J. Coil, H. Hurttia, H. Larjava, Oral fluid elastase as an indicator of periodontal health, J. Clin. Periodontol. 23 (1) (1996) 30-37.

[21] M. Zhou, H.X. Meng, Y.B. Zhao, Z.B. Chen, Changes of four proinflammatory proteins in whole saliva during experimental gingivitis, Chin. J. Dent. Res. 15 (2) (2012) 121-127.

[22] J. Travis, G.S. Salvesen, Human plasma proteinase inhibitors, Annu. Rev. Biochem. 52 (1983) 655-709.

[23] P. Skottrup, G. Dahlén, V. Baelum, R. Lopez, Soluble urokinase-type plasminogen activator receptor (suPAR) is associated with signs of periodontitis in adolescents, Eur. J. Oral Sci. 126 (4) (2018). 